This paper was presented at the Innovations in NGN: Future Network and Services, 2008. First ITU-T Kaleidoscope Academic Conference, Geneva, Switzerland May 13, 2008. It is Published in the proceedings of that conference and IEEE Xplore.

\title{
Exploring Adaptable Access in Next Generation Networks
}

\author{
Ken Krechmer \\ University of Colorado at Boulder \\ 757 Greer Road • Palo Alto, CA 94303-3024 \\ +1-650-856-8836・krechmer@csrstds.com
}

\begin{abstract}
An "etiquette" is required but is missing from the planned NGN architecture. An etiquette provides the mechanism to fairly select which access technology to use when multiple access technologies are deployed in the same network. This paper identifies the benefits an etiquette offers, describes how an etiquette can improve NGN over $3 \mathrm{G}$ networks, and explains how standardizing an etiquette for NGN can affect intellectual property issues.
\end{abstract}

\section{What's Missing?}

ITU-T Y.2012, Next Generation Networks - Functional requirements and architecture of NGN release 1, specifies the requirement to support multiple access technologies in NGN [1]. Similarly, ITU-T Y.2001, the overview of the Next Generation Networks, states that the user will have the ability to access available services [2].

These requirements are the same as those adopted in Berlin in 1906 [3]: "The coastal stations and the on-board stations must exchange radio telegrams irrespective of the radio-telegraphic system adopted by these stations."

After 100 years, the NGN requirements still do not address the users' and service providers' need to fairly select among multiple access technologies when more than a single access technology is available. It appears that the current documentation on NGN supports flexible access technology (selection without negotiation) but not adaptable access technology (selection after negotiation) [4]. On first glance this seems a small point. But adaptability has significant impacts on the performance and operational capabilities of any communications system and even on the standardization process itself. This paper focuses on wireless communications access systems as they are the most easily adaptable.

\section{Wireless Systems Today}

Different wireless access technologies may include several generations of cellular technologies (ITU-T IMT-2000, IMT-Advanced), several generations of wireless LANs 
utilizing Wi-Fi (IEEE 802.11a/b/g/n) and the wireless MAN communications technology WiMAX (IEEE 802.16 [recently included in IMT-Advanced], 802.20, 802.22). These technologies and national variations (e.g., wireless and cellular systems defined by the CCSA for use in China [5]) will occupy different frequency bands in different regions of the world (e.g., $700 \mathrm{MHz}$ in the US or $2.6 \mathrm{GHz}$ in some European countries are emerging wireless bands). In this paper, access technology refers to the protocols and signals below the Internet Protocol layer (sometimes termed an air interface), used to implement a specific communications technology. The implementation of different access technologies in the same system is termed a heterogeneous access system.

Real time processing, digital signal processors (DSP), cognitive radio (CR), software defined radio (SDR), and low power/cost semiconductor memory together make heterogeneous access systems practical. The new technologies of SDR and CR underlie dynamic spectrum sharing systems (DSSS) which promise to increase utilization of a broad radio spectrum by adapting to avoid spectral interference. One application of CR increases spectrum utilization among licensed and unlicensed users [6]. One application of SDR supports multiple air interface technologies. However, the SDR and DSSS approaches also suggest other opportunities to improve access systems that have not yet been explored. It may be useful to step back from the existing applications of SDR and DSSS technologies to examine the broader implications of heterogeneous access systems.

Utilizing the range of these technologies, it is possible to create access systems that can be compatible with different access systems. What is missing is a means and mechanism to identify how interoperation between communicating ends of the access system is to be achieved. Interoperation is defined as compatible operation, including data transfer, between two or more communications applications in autonomous terminals or nodes.

Standardizing heterogeneous communications systems is new for standardization organizations. Creating standards which support change is quite different from creating standards which minimize change. Determining the additional requirements needed to support heterogeneous communications requires a real paradigm shift in basic standardization goals and ideals held by standardization committees.

Previously, a clear goal of communications standardization has been to define a single communications technology or protocol at each appropriate OSI model layer (X.200/ISO7498). As example, the breath and success of the Internet is based on the ubiquitous use of the Internet Protocol. Heterogeneous access standardization requires a shift in the operation of standardization organizations from limiting change (which tends to force compatibility), to supporting change and creating the adaptive mechanisms to cope with the resulting heterogeneous environment. It will require wise and forward thinking leadership to accomplish such a paradigm shift in any standardization organization.

The current standardization effort most closely focused on communications adaptability is the End-to-End Reconfigurability $\left(\mathrm{E}^{2} \mathrm{R}\right)$ project. Phase I of this project proposed a Reconfiguration Management Plane as part of the systems architecture that supports 
heterogeneous communications [7]. Phase II proposes support for autonomic operation (self-configuring, self-healing, self-optimizing and self-protecting) utilizing in-network resources [8]. The $E^{2} R$ project focuses on developing the architecture for adaptable systems but does not address the unique aspects of adaptability in the access network. The architecture proposed in the $E^{2} \mathrm{R}$ project and in this paper have much in common and also some salient differences, which are discussed below.

\section{Adaptability}

The term adaptability is used in this paper to describe how interoperation is created and maintained between autonomous heterogeneous systems at all layers of the OSI (X.200) model. A system that is adaptable includes three processes: identification, negotiation and selection prior to data or control transfer. Identification provides a listing of the distinct choices available and/or possible at each communicating end to the other end. Identification examines parameters which define a specific spectrum in time, power, frequency and/or coding and also identifies specific protocols or options (at any communications layer) to determine characteristics of the communications system. Negotiation refers to the process of determining which specific characteristics of the lists of identified transmitter and receiver end characteristics are to be utilized for communications. Negotiation then implements the logic (which may exist in remote repositories) necessary to identify the most desired variation common to each communicating end. Selection chooses the desired common communications mechanisms for data and control information. Selection also includes the installation (if required) and/or initialization of the selected parameters to allow compatible communications to begin.

\section{Current Variations of Adaptability}

Adaptability may be accomplished using independent computers (e.g., personal computers) in end nodes connected via a network. Such end nodes are capable of autonomous operation relative to each other and to the network. This is the architecture that the Internet uses. Flexibility may be accomplished using multi-mode cell phones that select the latest generation technology and strongest signal available. This is the architecture that existing cellular networks use. Heterogeneous access networks for NGN require using aspects of both architectures.

Flexibility is a sub-set of adaptability. Flexibility, a one-sided way to select a specific technology, may be achieved using a gateway. A simple gateway example is an Edison light bulb socket which supports many different types of light bulbs. In this example the human user identifies the specific light bulb, while the Edison light bulb socket (the gateway) makes this selection possible. Such a gateway only supports selection from the user side as the gateway is fixed.

Another example of flexibility is offered by the meta-protocols based on XML. These meta-protocols support identification of resources but leave negotiation and selection to other processes. The Internet Protocol is a communications protocol that acts as a 
spanning layer (a gateway across space) [8]. Negotiation mechanisms are not defined in the Internet Protocol, but can exist in protocols that assume an Internet Protocol datagram capability.

Service discovery protocols are an example of protocols that look for additional services above the IP layer. Examples of service discovery protocols include Jini for Java objects, Universal Plug-and-Play (UPnP) based on the IETF Simple Service Discovery Protocol (SSDP), Service Location Protocol (SLP) standardized by the IETF as RFC 2608, and Bluetooth Service Discovery Protocol (SDP) [9]. These service discovery protocols support the identification and selection functions and could be expanded to support negotiation. However, such protocols cannot identify, negotiate or select lower layer services such as physical or data link layer services.

Another protocol example, IETF RFC 2543, Session Initiation Protocol (SIP) supports adaptability for multimedia communications above the Internet Protocol layer.

Other standardization organizations that are actively developing adaptability standards include: IEEE 802.21 is developing standards to enable handover and interoperability between heterogeneous network types including both 802 and non-802 networks. The IETF Control And Provisioning of Wireless Access Points (capwap) working group is developing mechanisms to support 802.11 wireless technologies. The 3rd Generation Partnership Project (3GPP) brings together a number of telecommunications standardization bodies which are known as "Organizational Partners." The current Organizational Partners are ARIB, CCSA, ETSI, ATIS, TTA, and TTC. 3GPP TS 23.002 V8.1.1 (2007-10) is the Technical Specification Group Services and Systems Aspects; Network architecture (Release 8) and includes 3GPP/WLAN interworking. Neither of these organizations are considering etiquettes.

Autonomic computing systems support adaptability with three processes: discovery, policy management (with related registries), and reconfiguration [10]. These three autonomic process closely relate to the processes of identification, negotiation and selection but support broader functionality. For reasons developed below adaptability for a communications system is more narrowly defined.

The term adaptability has been used in software development to refer to upgradeability, individualization, or changes associated with different operating environments. Sometimes such approaches are termed open source programming. Open source programming may improve the adaptability of software programs by enlisting additional programmers on an ad hoc basis. Such usage of the term adaptability is only addressed indirectly here.

Even heterogeneous communications systems must be defined by standards to allow compatible operation. The standardized aspects of communications systems are not appropriate for the ad hoc processes that characterize open source development. The adaptability of communications systems proposed in this paper must be controlled via standardization, and perhaps certification processes, or interoperation will suffer. But it 
seems reasonable to suggest that adaptable communications systems are more open and that standards that support adaptable communications are more open standards [11].

The End-to-End Reconfigurability $\left(\mathrm{E}^{2} \mathrm{R}\right)$ project recognizes the Y.2012 requirements; it proposes that interoperation be supported by a network-based policy management system and in-network registries that maintain the database of all distinct options supported on each specific mobile terminal. The policy management system also includes the logic to decide how best to interconnect heterogeneous mobile terminals, networks and service providers. However the $\mathrm{E}^{2} \mathrm{R}$ approach to adaptability does not specifically address how to achieve autonomous operation of the end nodes and terminals. Achieving autonomous operation down to the physical layer requires an etiquette.

\section{Etiquettes}

An etiquette is a set of protocols (including the physical layer functions) which does not pass data or control but only identifies, negotiates and selects the ability to pass data or control. An etiquette becomes a common denominator which shuttles back and forth between the communicating ends to negotiate which specific access technologies, protocol(s), data sets and options will be used over a link, band or set of bands where multiple different standards, options and/or proprietary versions exist. An etiquette utilizes an independent, bidirectional communications channel, potentially operating at a much lower data rate than a main or control channel. Simulations, which project near optimum bandwidth utilization, using a very simple wireless etiquette are shown in [12].

High speed programmable processors with changeable memory in each node or terminal provide the means to implement basic communications yet adapt to utilize new communications programs and allow proprietary communications technology. Once all communications functions are programmable and changeable, they can be adapted, to support any new communications invention (within the constraints of the resources available) and still allow backward compatible operation.

The purpose of an etiquette is to determine which access technologies are available, compatible and mutually desirable before any transfer of data or control information occurs. While an etiquette may support any of the layers of the OSI communications model, we propose that etiquettes for NGN provide identification, negotiation and selection functionality below the Internet Protocol layer. This layered differentiation keeps the underlying transport technologies independent from the service-related functions and leaves identification, negotiation and selection above the IP layer to existing or new protocols (e.g., SIP or discovery protocols). This layering approach also follows the model supported by 802.21 and the IETF.

In the OSI model, an etiquette represents a specific range of functions within the X.200 management stack. An etiquette may also be seen as part of the $\mathrm{E}^{2} \mathrm{R}$ project's proposed Reconfiguration Management Plane.

\section{Existing Etiquettes}


Group 3 facsimile uses a 300 bit/s channel (defined in ITU-T T.30), which negotiates the operation of the higher rate data and control channel defined in T.4. T.30 is an example of an etiquette. This etiquette has been very successfully extended (e.g., supporting facsimile date rates of 4800, 9600, 14400 and $28800 \mathrm{bit} / \mathrm{s}$ ) for over thirty years.

Supporting long term compatibility greatly contributed to the overall term success of G3 facsimile. Even where incorrect new G3 implementations occurred, fallback to reduced, but compatible, operation was possible.

Other existing examples of etiquettes used to negotiate with remote systems include the International Telecommunications Union (ITU) V.8 used by voice-band modems to negotiate remote compatible operation with the far-end modem. Initially wireline modem standards (ITU-T V.21, V.22, V.23, V.22bis, V.32 and V.32bis) did not use an etiquette. However, as the number of modem standards that needed to be identified increased, the reliability of the identification decreased and it eventually was recognized that an etiquette was needed. Now ITU-T modems V.34, V.90, V.91 and V.92 are all designed to use the ITU-T V.8 etiquette. As an example of how V.8 allowed the introduction of a new capability into older modems, in November, 2000, a new error control protocol (V.44) optimized for Internet transfers was added to these older modem standards with the addition of a specific V.44 identifier in V.8.

The ITU-T Digital Subscriber Line (DSL) standards use an etiquette (G.994.1) which maintains backward compatibility with the earlier North American DSL standard T1.413 and supports compatibility with other DSL standards (as of Feb. 2007: G.991.2, G.992.1, G.992.2, G.992.3, G.992.4, G.992.5, G.993.1 and G.993.2).

It is worth noting that each of these etiquettes has been very successful in maintaining compatibility of new and evolving technologies in very large systems ( $>10^{8}$ terminals), over long spans of time ( $>10$ years) implemented by many different developers $(>100)$.

\section{The Value of Etiquettes for NGN}

$3 \mathrm{G}$ and earlier cellular systems currently do not utilize an etiquette. The IMT-2000 series of standards do not define any mechanism to select among the five different physical layer technologies defined and their corresponding protocol stacks. An existing $3 \mathrm{G}$ cellular mobile may listen for the different control channel signals in multiple bands using different technologies. This level of flexibility is termed multi-mode operation. The multi-mode 3G mobile/system selects the appropriate band and technology for user communications based on a proprietary approach. There is no back-and-forth negotiation between the terminal and the tower.

Utilizing an etiquette offers at least 12 advantages over the approach taken in the $3 \mathrm{G}$ system:

1. The range of signal strength and interference in an operating $3 \mathrm{G}$ system means that different receivers (one or more $1 \mathrm{G}, 2 \mathrm{G}$ and $3 \mathrm{G}$ technologies) may not reliably find a 
compatible transmitter. Alternatively, a multi-mode receiver can identify a compatible transmitter in all cases, but this may require a smaller served area and/or a longer time to establish the connection.

2. Current approaches to fixed-mobile convergence do not support etiquettes in either the fixed or mobile bands so it is not possible to choose when switching between fixed and mobile bands which of the multiple standards available is the most desirable [13].

3. An etiquette supports changing to a better performing technology from among the different air interface technologies available. This increases the multi-mode system's functional area.

4. An etiquette offers another means to avoid spectral interference that introduces very little delay in the start of data or control communications.

Points 1, 2, 3, and 4 support increased system capacity. A low data rate etiquette that uses an independent band selected to have the lowest noise provides a more robust transmission technology than the control or communications air interfaces it is designed to negotiate. This is a very desirable means to support some level of communications when there is high noise or low received signal.

Cell area size, communications start-up time, and spectral interference are dependent on both terrain and system. In general, the more air interface technologies supported in the same band (fixed or mobile), the more positive is the effect of using an etiquette on geographic coverage, start-up delay and/or interference. For this reason, etiquettes will be most advantageous in links or bands not limited to a single standard (e.g., US industrial, scientific and medical [ISM] radio bands). It is possible to use etiquettes to identify known interfering transmitters. This provides an enhancement to existing cognitive radio concepts [6].

5. The multi-mode receiver does not know which of the multi-mode transmitter technologies may exist in a local multi-mode transmitter system. Thus the receiver will not always select the most desirable transmitter/receiver pair.

6. Negotiation makes it possible to balance the desires of the user and service provider. As example, in a $3 \mathrm{G}$ cellular system the user (cell phone end) may desire the lowest cost service, while the service provider (base station end) may desire the lightest loaded service. The lowest cost service is not likely to be the lightest loaded service and so service selection may require logic based on the users' contract with the service provider.

Points 5 and 6 address the desirability of negotiation in allowing user and/or service provider needs to be supported. Negotiation is the most difficult adaptability process to define as each communicating end may have different priorities. Negotiation is also identified as a requirement in the $E^{2} R$ project documents. Most existing etiquettes utilize a priority structure based on the idea that more is better. In other words, the highest data rate, most dense picture format, largest picture format, broadest range of colors, etc. that is common to both communicating ends is treated as most desirable. NGN systems will 
likely require more complex negotiations. The $E^{2} R$ project documents suggest the use of a policy repository and policy management system to address more complex negotiations.

7. An etiquette allows a negotiation and fallback when an implementation in a specific mode is found not to be compatible. This reduces the operational costs and increases the service life (by supporting easier upgrades) of each programmable system component.

8. The Software Defined Radio concept is likely to increase long-term compatibility issues, making etiquettes more necessary.

Points 7 and 8 address maintaining compatible system operation over longer spans of time. Changes to access systems (new implementations as well as standards revisions) may decrease total system compatibility. Verification of compatible operation of multilayer, multi-mode implementations is problematic as the number of possible combinations to test becomes very large. Supporting compatible operation over long spans of time may be economically very significant to both users and service providers. The use of etiquettes to maintain long term compatibility in heterogeneous access systems is not addressed in the $\mathrm{E}^{2} \mathrm{R}$ documentation referenced.

9. An etiquette offers a technology-neutral means to negotiate all available control and/or main channel access technologies.

10. Without an etiquette, proprietary technologies are either excluded or required in a specific access technology standard. Too often, standardizing an access technology creates a patent-holder-wins standard.

11. Without negotiation, the standardization committee selects the winning proprietary access technologies. With a negotiation, the market can select the winning proprietary access technology.

These three points address how etiquettes can impact standardization committees when intellectual property is involved. Etiquettes are based on older technology (because the etiquette channel requires low data rates) thereby minimizing intellectual property issues associated with an etiquette. This avoids the contentious issues associated with picking a single data or control channel technology (with associated intellectual property rights) to support negotiation.

Since an etiquette can negotiate private as well as public access technologies, features and options, the standardization committee does not have to make such choices. Standardization committees can require that any private intellectual property be supported only as a proprietary feature unless the committee believes that the private intellectual property supports functionality so cost effective that it should be included in a public standard. Where proprietary technology is included in a public standard, the standardization committee should require different approaches to the same function in the same standard so as to ensure there is competition. Supporting market driven technology represents a new approach to standardization committee operation. 
12. When no compatible data or control channel communications is identified between communicating ends, the parameters transferred by the etiquette provide the exact details to each end of what is necessary to make communications possible.

Point 12 is very significant in simplifying the use and maintenance of very large heterogeneous systems. With an etiquette, it is possible for either communicating end, when incompatibility is identified, to automatically use alternative networks to download the needed access technology to make interoperation possible. As example, the base station could download from the Internet the access technology needed for interoperation with a new remote terminal.

As previous experience with telephone modems has shown, it is practical to start initially without an etiquette, then standardize the etiquette before the multiplicity of access technologies becomes too cumbersome. An etiquette for NGN could be implemented in parallel with existing IMT-2000 selection mechanisms. When the etiquette is available, it should be used, otherwise the previous proprietary (and more limiting) selection mechanisms are used. It may also be possible to software upgrade some IMT-2000 implementations to add the etiquette functionality.

\section{Structure of an Etiquette}

Understanding the operation of an etiquette and why it must be implemented separately requires a look at the etiquette's logical structure. Etiquettes must be designed as a single tree structured logic to ensure that all revisions of the etiquette remain completely backward compatible. Using a single, unambiguous tree structure ensures that additions to the etiquette are always a logical super set (includes exactly ALL the previous capabilities plus the addition, deletions are not allowed). This comes as close as is possible to ensuring that each change in the etiquette is backward compatible. This concept is not addressed in the $\mathrm{E}^{2} \mathrm{R}$ documentation referenced.

If one could be certain that all changes to an etiquette were always a proper super set, then an etiquette would not require revision control. Because of possible flaws in etiquette receiver design (e.g., not a large enough receive buffer), this may not practical. This does suggest that the etiquette receiver in a communications system be tested to verify that it ignores what it does not understand (revisions to the etiquette newer than the receiver) and that it does not have buffer overflow issues. This form of testing is not usually done in current communications system testing or certification.

To prevent any possible communications incompatibility, an etiquette must transmit a revision level to allow backing off to the previous revision when etiquette revisions are made that are not fully compatible with the previous version. Since, in the worst case, systems desiring to be fully compatible would need to support all etiquette revision levels, additions to etiquette functionality (new revision levels) should be made with great care. The maintenance of etiquette standards also requires more care than compatibility standards. Etiquette standards will be used for far longer periods than the compatibility standards they negotiate. 
The access system's data and control channels are more likely to change, more likely to be time dependent (therefore more difficult to test for compatibility), or require deletions, thus maintaining backward compatibility without an etiquette becomes almost impossible as the possible variations become large enough. Using an etiquette to identify, negotiate and select operational functionality is a much more reliable means to maintain long-term compatibility than expecting the data or control channels to maintain compatibility. The Resource Management Plane appears to address a similar issue in the $E^{2} R$ project.

A proper etiquette contains fields defining the etiquette revision level, a separate branch for each technology and protocol supported with revision level, options available and possibly a priority level of each (where the priority is not established by a simple sequence or remote process). Adding new protocol branches to the etiquette allows the support of additional protocols without affecting the compatible operation of existing protocols. As example, the T.30 etiquette in Group 3 facsimile has been used for over 30 years while the compatibility standards (modems) defined in T.4 have changed at least four times over the same period.

\section{Etiquettes Support Proprietary Enhancements}

Etiquette standards offer new ways to implement, control and add value to access systems. The negotiation supported by an etiquette can identify any possible compatibility, including support of proprietary enhancements using a standardized way of passing proprietary information. When a proprietary enhancements field is utilized, it begins with a unique and proprietary code identifying the owner of the proprietary enhancement. After the unique and proprietary code, the serial information structure is determined by the owner(s) of the proprietary enhancement.

The unique and proprietary code may be used to maintain ownership control. In Group 3 facsimile, the standardized way to support proprietary enhancements is called ITU T.30 Non-Standard Facilities (NSF). Each NSF is identified by a unique information sequence: ITU country code (T.35), manufacturer's unique code (registered by a national organization) and then any private information identifying proprietary enhancements may be exchanged. This permits communicating ends which have identified each other to then exchange proprietary information. In a higher layer adaptability mechanism, Session Initiation Protocol, a reverse order domain name is used to provide the unique identity. Other examples of ways to provide the necessary unique identity currently in use include: ASCII representation of trade names, Internet domain names and the ASCAP (American Society of Composers, Authors and Publishers) five bars of music.

The different unique and proprietary codes, including those in T.30 NSF and SIP, may be considered owned by the organization that implements them, and there is precedent to suggest that that such ownership is legally recognized. These information sequences become the identification that communicating systems use to verify design ownership. By using the unique and proprietary code, the communications equipment manufacturer/developer maintains legal control over any proprietary features that are 
enabled or transferred after the unique and proprietary code. After a time of proprietary success, desirable proprietary enhancements may become public by the standardization of similar functions in the etiquette's public parameter sets.

The use of a unique and proprietary code to support proprietary enhancements in public standards has been quite successful for some companies. Ricoh, a Japanese facsimile machine manufacturer, offered proprietary higher speed G3 facsimile to its corporate customers. Then, a few years later, higher speed operation similar to what Ricoh pioneered was included in the G3 public facsimile standard. This transition occurred several times. Capitalizing on this approach, Ricoh became the largest provider of G3 facsimile machines to the corporate market in the 1980's and 1990's.

Etiquettes, via the unique and proprietary code, open the possibility of a different business model for wireless service providers. Currently many service providers attempt to control their markets via long term contracts or mechanisms that lock the user's cell phone to a specific service provider. Offering proprietary features negotiated via etiquettes, service providers could control specific markets based on their innovation, without unfairly controlling the user. For example, the banking industry may want stronger encryption, the radiology market may want higher resolution, the wireless data market may want better error control. As service providers offer unique communications features, they can add them to the proprietary enhancements field, as Ricoh did with higher speed facsimile. In this manner, service providers can add value yet support open standards.

Supporting a mechanism to identify proprietary features above or below the Internet Protocol layer is not addressed in the ITU or $E^{2} \mathrm{R}$ documentation referenced. This eliminates new and fairer business models where manufacturers, developers and service providers may be compensated for their innovation directly by their markets. Not supporting proprietary enhancements appears to be a significant omission in the documentation on NGN, whether or not etiquettes are used.

In summary, the properties of an etiquette for NGN include:

- Below Internet Protocol layer support.

- Negotiation services without operational functionality.

- Single tree, unambiguous, logical structure.

- Deletions are not allowed.

- An etiquette receiver ignores what it does not understand.

- Mechanism available to prioritize each branch.

- Supports proprietary functionality.

- Etiquette revision level.

\section{Making NGN Adaptable}

Adaptability can address multiple issues that bedevil heterogeneous communications systems: 
- Supporting the rapid introduction of new technology.

- Supporting proprietary, national and regional functions and features within public standards.

- Supporting negotiation of modes, features and options.

- Selecting compatible modes between different multi-mode systems while increasing system capacity.

- Maintaining interoperation between multiple revisions of standards.

- Maintaining interoperation between different implementations of the same technology or protocol.

- Moves intellectual property issues to the market.

- Identifying the specific reason(s) when interoperation fails.

- Avoiding interfering communications.

There are a number of different technical approaches to adaptability including: autonomic computing, meta-protocols, open source, service discovery, and spanning layers. While each of these approaches offers valuable insights into NGN requirements, none address all the requirements of heterogeneous access systems.

Work on adaptability is occurring in many standardization organizations including the IEEE, IETF, E ${ }^{2}$ R, FMCA and 3GPP. Each organization tends to focus on its own needs without a recognition that adaptability is more than flexibility, is more complex (not only network selection but also revision and implementation interoperation) and more general (across all networks). There is a need for more complete requirements and more coordination among all involved standardization organizations.

A hundred years after the first International Radiotelegraph Conference, NGN should offer greater capabilities to the users and service providers than were supported in 1906. This can be accomplished by standardizing adaptable processes. Standardization leadership will be necessary to make this happen. The ITU is the communications standardization organization with prior experience developing etiquettes, active in spectrum allocation (needed for wireless etiquette channels), the leader in IMT standardization, a long history and world-wide membership. If the ITU can respond to the challenge that adaptability standardization represents, then it is the most appropriate standardization organization to develop etiquettes for IMT-Advanced and coordinate the full range of adaptability requirements for NGN.

\section{References}

[1] ITU-T Y.2012 (09/2006) Next Generation Networks - Functional requirements and architecture of NGN release 1 .

[2] ITU-T Y.2001 (12/2004) Next Generation Networks - General overview of NGN.

[3] This quotation (original in French) is in Article 3 of the annex to the International Radiotelegraph Convention adopted at the first International Radiotelegraph Conference, a predecessor of the ITU-R, Berlin, 1906. 
[4] Network Architecture and Support Services for Reconfigurability, Wireless World Research Forum, Working Group 6, White Paper, December, 2004, http://e2r.motlabs.com/dissemination/whitepapers

[5] http://www.cwts.org/english/ Quoting from this web site: "To establish a nationwide unified communications standardization organization that is compatible with international norms and Chinese situations and capable of meeting the needs in the market, a number of senior experts, among them Mr. Wei Leping and Mr. Wu Hequan, proposed to establish China's communications standard organization on the basis of existing standard working groups. With the approval of MII and Standardization Administration of China and registered in the Ministry of Civil Affairs, China Communications Standards Association was established on 18 December 2002."

[6] Sirinivasa, Sudhir \& Syed Ali Jafar The Throughput Potential of Cognitive Radio: A Theoretical Perspective, IEEE Communications Magazine, Vol. 45 No. 5, May 2007 pp. 73-79.

[7] The End-to-End Reconfigurability $\left(E^{2} R\right)$ project of the 6th Framework Programme of the European Commission, Network Architecture and Support Services for Reconfigurability, Phase I Working Group 6 White Paper, undated, available via http://e2r.motlabs.com/front-page

[8] Clark, David D., "Interoperation, Open Interfaces, and Protocol Architecture," 1995 http://bob.nap.edu/html/wpni3/ch-16.html

[9] Richard, Golden G., Service and Device Discovery Protocols and Programming, McGraw-Hill, New York, NY, 2002.

[10] Evolution of Reconfiguration Management and Control System Architecture, E2R II Deliverable D2.1, August 7, 2006, available via http://e2r2.motlabs.com/Deliverables

[11] For a discussion of the differences between open source and open standards see: Krechmer, K., Cathedrals, Libraries and Bazaars, Proceedings of the 17th Symposium on Applied Computing (SAC), ACM Press, 2002, p. 1053-1057. Available at http://www.csrstds.com/cathedrals.html

[12] R. Garces, J.J. Garcia-Luna-Aceves, and R. Rom, An Access Etiquette for VeryWide Wireless Bands, IEEE IC3N'98, Lafayette, Louisiana, October 12-15, 1998. Available at http://citeseer.ist.psu.edu/607714.html

[13] The Fixed-Mobile Convergence Alliance (FMCA) was formed in June 2004 and incorporated as a not-for-profit trade association under New York law in August 2006. http://www.thefmca.com/ 